\title{
The Effectiveness of Pemetrexed Monotherapy Depending on Polymorphisms in TS and MTHFR Genes as Well as Clinical Factors in Advanced NSCLC Patients
}

\author{
Tomasz Kucharczyk ${ }^{1,2}$ • Pawel Krawczyk ${ }^{1}$ • Tomasz Powrózek ${ }^{1}$ - Dariusz M. Kowalski ${ }^{3}$ • \\ Rodryg Ramlau ${ }^{4,5}$ • Ewa Kalinka-Warzocha ${ }^{6}$. Magdalena Knetki-Wróblewska ${ }^{3}$. \\ Kinga Winiarczyk ${ }^{3}$ - Maciej Krzakowski ${ }^{3}$. Janusz Milanowski ${ }^{1,7}$
}

Received: 20 November 2014 / Accepted: 3 August 2015 / Published online: 16 August 2015

(C) The Author(s) 2015. This article is published with open access at Springerlink.com

\begin{abstract}
In NSCLC, second-line chemotherapy using pemetrexed or docetaxel has limited efficacy and should be dedicated to selected groups of patients. Pemetrexed is an antifolate compound with the ability to inhibit enzymes (TS, DHFR and GARFT) involved in pyrimidine and purine synthesis. The objective of this study was to evaluate the association between polymorphisms of TS and MHFR genes and clinical outcomes in NSCLC patients treated with pemetrexed monotherapy. DNA was isolated from peripheral blood of 72 non-squamous NSCLC patients treated with pemetrexed. Using PCR and RFLP methods, the variable number of tandem repeats (VNTR), the G $>$ C SNP in these repeats and insertion/deletion polymorphism of TS gene as well as $677 \mathrm{C}>\mathrm{T}$ SNP in MTHFR gene were analyzed and correlated
\end{abstract}

Paweł Krawczyk

krapa@poczta.onet.pl

1 Department of Pneumonology, Oncology and Allergology, Medical University of Lublin, Jaczewskiego 8, 20-954 Lublin, Poland

2 Postgraduate School of Molecular Medicine, Warsaw Medical University, Żwirki i Wigury 61, 02-091 Warszawa, Poland

3 Department of Lung and Chest Cancer, Oncology Centre-Institute, M. Sklodowska-Curie in Warsaw, W. K. Roentgena 5, 02-781 Warszawa, Poland

4 Greater Poland Center of Pulmonology and Thoracic Surgery of Eugenia and Janusz Zeyland, Poznań, Poland

5 Department of Clinical Oncology, Chair of Cardio-Thoracic Surgery, University of Medical Sciences, Szamarzewskiego 82/84, 60-569 Poznań, Poland

6 Regional Centre of Oncology in Łódź, Ignacego Paderewskiego 4, 90-993 Łódź, Poland

7 Institute of Agricultural Medicine of Lublin, Kazimierza Jaczewskiego 2, 20-950 Lublin, Poland with disease control rate, progression-free survival and overall survival (OS) of NSCLC patients. Carriers of $2 R / 3 R(G)$, $3 R(C) / 3 R(G), 3 R(G) / 3 R(G)$ genotypes showed significantly more frequent early progression than carriers of $2 \mathrm{R} / 2 \mathrm{R}, 2 \mathrm{R} /$ $3 \mathrm{R}(\mathrm{C}), 3 \mathrm{R}(\mathrm{C}) / 3 \mathrm{R}(\mathrm{C})$ genotypes of $T S$ gene $(p<0.05)$. Among carriers of triple $28 \mathrm{bp}$ tandem repeats (3R) in $T S$ gene and $\mathrm{C} /$ C genotype of MTHFR gene a significantly shorter OS was observed ( $\mathrm{HR}=3.07 ; p=0.003)$. In multivariate analysis, significantly higher risk of death was observed in carriers of both $3 \mathrm{R} / 3 \mathrm{R}$ genotype in $T S$ and $\mathrm{C} / \mathrm{C}$ genotype in $677 \mathrm{C}>\mathrm{T}$ SNP in MTHFR (HR $=3.85 ; p<0.005)$ as well as in patients with short duration of response to first-line chemotherapy $(\mathrm{HR}=2.09 ; p<0.005)$. Results of our study suggested that genetic factors may have a high predictive and prognostic value (even greater than clinical factors) for patients treated with pemetrexed monotherapy.

Keywords Non-small cell lung cancer $\cdot$ Pemetrexed monotherapy $\cdot T S \cdot M T H F R \cdot$ Polymorphism

\section{Introduction}

Lung cancer is the most prevalent cause of death due to malignancies worldwide. There is very low percentage of early diagnosed NSCLC patients which results in only $15 \%$ chance of surgical resection [1]. Systemic therapy (chemo- and radiotherapy) is considered to be the main form of lung cancer treatment. Unfortunately, first-line treatment is of low effectiveness and progression is observed in the vast majority of patients.

Pemetrexed is one of the novel 3rd generation drugs with the least side effects reported. In the first-line study, cisplatin with pemetrexed proved to be more effective in non- 
squamous histology of NSCLC and as a result it was registered in only such histology of lung cancer [2]. Second-line treatment with pemetrexed monotherapy is possible in nonsquamous patients with good performance status who progressed after non-pemetrexed first-line chemotherapy, which was proven by a comparative study with docetaxel [3].

Pemetrexed, a multitarget antifolate, is effective in nonsquamous NSCLC and malignant pleural mesothelioma, with the main focus on inhibition of enzymes involved in pyrimidine and purine synthesis such as thymidylate synthase (TS), dihydrofolate reductase (DHFR) and glycinamide ribonucleotide formyltransferase (GARFT). TS catalyzes transformation of dUMP into dTMP. Decreased levels of dTMP results in inhibition of DNA repair and synthesis, thus cell death [2-4].

Studies have shown that expression levels of pemetrexed target enzymes may alter the effectiveness of the drug [5, 6, 7]. Squamous cell carcinoma was shown to have higher expression of TS than adenocarcinoma, thus being more resistant to pemetrexed treatment $[8,9]$.

Free circulating folates may regulate pemetrexed targets' activity. Increased level of methyltetrahydrofolate (5methylTHF) is a result of changes in the activity of 5,10methylenetetrahydrofolate reductase (MTHFR), which results in higher TS activity and thus reduces pemetrexed efficacy [10].

There are three well known polymorphic changes that affect TS mRNA level and TS protein expression: various number of 28-base pair tandem repeats (VNTR) located in the $5^{\prime}$ end untranslated region of TS gene; a single nucleotide polymorphism (SNP) $\mathrm{G}>\mathrm{C}$ in the second repeat of the 28-bp tandem repeats; and a 6-bp deletion at the 3' end of the TS gene (1494del6) [11-13]. Polymorphisms in TS gene are known to alter effectiveness of 5-fluorouracil (5-FU) in colorectal cancer [14]. Moreover, MTHFR gene polymorphism $677 \mathrm{C}>\mathrm{T}$ is causing lower expression of MTHFR and decreased levels of 5-methylTHF in colon and breast cancer cell lines [15].

This retrospective, non-randomized, multicenter study was carried out in order to assess the usefulness of TS and MTHFR gene polymorphisms as predictive markers in NSCLC patients treated with pemetrexed monotherapy.

\section{Materials and Methods}

The whole studied group consisted of 72 NSCLC patients (46 male, 26 female; median age 61) with non-squamous histology, who were treated with pemetrexed monotherapy. Clinical data was collected from all patients. $500 \mathrm{mg} / \mathrm{m}^{2}$ of pemetrexed was administered as an intravenous infusion on day 1 of each 21 day cycle. In order to reduce toxicity, patients received folic acid and B12 vitamin prior to treatment.
The response to chemotherapy was assessed according to RECIST criteria. The observation period was from 2008 until February 2014, when progression was observed in 60 patients of whom 45 died.

The material for the study were blood samples collected in four oncology centres in Poland. All genetic testing was performed in clinical laboratory in Pneumonology, Oncology and Allergology Department in Lublin. Venous blood samples of $5 \mathrm{ml}$ have been collected to EDTA-covered tubes. DNA was extracted using QIAamp DNA Mini Kit (Qiagen, Germany).

The study was approved by the Ethics Committee of the Medical University in Lublin (No. KE-0254/219/2010).

\section{Genotyping of TS and MTHFR}

All studied polymorphism were analysed by polymerase chain reaction (PCR). Primers used in TS gene VNTR polymorphism analysis were previously described by lacopetta et al. [16]. The analysis of G $>C$ SNP in tandem repeats of TS gene was carried out using allele-specific PCR (ASP-PCR) with forward primers designed specifically for $\mathrm{G}$ or $\mathrm{C}$ nucleotide $\left(5^{\prime} \mathrm{CGTCCC}\right.$ GCCGCGCCACTTG 3' and 5'CGTCCCGCCGCGCCACT TC 3', respectively). The reverse primer was the same one used in the VNTR analysis, described by lacopetta et al. The ASPPCR was carried out in two separate PCR tubes, one for Gending primer and one for $\mathrm{C}$-ending primer. The 1494del6 deletion on the 3' end of TS gene and MTHFR 677C > T SNP were detected by restriction fragment length polymorphism (RFLP) technique. Primers used for the detection of 1494del6 were previously described by Dotor et al. [17], while the MTHFR $677 \mathrm{C}>\mathrm{T}$ SNP primers were previously described by Frosst et al. [18]. For the RFLP reaction Fermentas FastDigest Dral (Thermo Scientific, USA) for TS 1494del6 polymorphism and Fermentas FastDigest HinfI (Thermo Scientific, USA) for MTHFR $677 \mathrm{C}>\mathrm{T}$ SNP restriction enzymes were used. The digestion process was carried out under the following conditions: $10 \mathrm{~min}$. incubtion in $37^{\circ}$ followed by $5 \mathrm{~min}$. digestion in $65^{\circ}$.

Every polymorphism analysis was carried out in the same reaction mixture of $20 \mu \mathrm{l}$, with only PCR primers being changed specifically for the polymorphism. The mixture contained: $100 \mathrm{ng}$ of sample DNA, $1 \mu \mathrm{M}$ of each primer, $0.2 \mathrm{mM}$ of each dNTP, $2.4 \mathrm{mM}$ of $\mathrm{MgCl}_{2}$ and $1 \mathrm{U}$ Taq polymerase with $1 \times$ Reaction Buffer (Thermo Scientific, USA). All reactions were carried out in a Biometra TPersonal thermocycler (Biometra, Germany).

Products of PCR were visualised on $2 \%$ agarose gel with ethidium bromide. TS VNTR polymorphism PCR products differed according to the number of 28 bp repeats. In Caucasian population the most often described genotypes consist of two or three repeats: $2 \mathrm{R} / 2 \mathrm{R}$ and $3 \mathrm{R} / 3 \mathrm{R}$ homozygotes, and $2 \mathrm{R} / 3 \mathrm{R}$ heterozygotes. $2 \mathrm{R} / 2 \mathrm{R}$ homozygotes produce a $116 \mathrm{bp}$ band on the gel, 3R/3R homozygotes produce a 144 bp band, and heterozygotes show both bands - 116 and 
$144 \mathrm{bp}$. The ASP-PCR for the G > C SNP in TS gene shows two band patterns: the common $\mathrm{G}$ nucleotide presented three bands: 124 and $96 \mathrm{bp}$ specific for G-ending primer and $68 \mathrm{bp}$ band specific for $\mathrm{C}$-ending primer, the rare $\mathrm{C}$ nucleotide presented three bands of 124 bp for G-ending primer and two bands of 96 and $68 \mathrm{bp}$ for $\mathrm{C}$-ending primer. The reaction products of $\mathrm{G}>\mathrm{C}$ SNP are shown in two separate lanes, one for $\mathrm{G}$-ending primer and one for $\mathrm{C}$-ending primer.

The 6 bp deletion at the 3 ' end of TS gene shows two band types: 142 bp for $-6 /-6$ genotype and 148 bp for $+6 /+6$ bp. The PCR products digestion with DraI restriction enzyme is possible only when the insertion genotype is present, so it creates two smaller bands of 88 and $60 \mathrm{bp}$. The heterozygous genotype $+6 /-6$ presents four bands of $148,142,88$ and $60 \mathrm{bp}$. The products of PCR for $677 \mathrm{C}>\mathrm{T}$ SNP in MTHFR gene present following patterns: 198 bp band for $\mathrm{C} / \mathrm{C}$ genotype and 175 and 23 bp band for T/T genotype when digested with HinfI restriction enzyme. The $\mathrm{C} / \mathrm{T}$ heterozygote is present on the gel as three band of 198, 175 and $23 \mathrm{bp}$. The smallest band of $23 \mathrm{bp}$ is not visible on the gel as it is migrating on the gel with reaction primers.

\section{Statistical Analysis}

Yates' chi-square test was used to assess differences between categorical variables and deviation from the Hardy-Weinberg equilibrium. Groups of patients with different clinical and molecular factors were compared using Kaplan-Meier method, in order to determine the probability of progression-free survival (PFS) and overall survival (OS). To determine the influence of the studied factors on PFS and OS of patients treated with pemetrexed monotherapy, the Cox regression model with step-by-step selection was used.

\section{Results}

The studied group consisted of NSCLC patients treated with pemetrexed monotherapy in second- or third-line and first-line patients who were not eligible for cisplatin. In this group there were 24 carriers of double $28 \mathrm{bp}$ tandem repeats (33.3\%), 17 triple repeats $(23.6 \%)$, the rest $(43.1 \%)$ were heterozygous. Among the carriers of triple repeats in one or both alleles, the $\mathrm{G}$ nucleotide in the second repeat of 28 bp appeared in 25 patients $(34.7 \%)$. The presence of 1494del6 deletion was observed in 8 patients $(11.1 \%)$, there were 28 carriers of one allele with deletion $(38.95 \%)$ and the insertion was present in $50 \%$ of patients. Allele T in $677 \mathrm{C}>\mathrm{T}$ SNP in MTHFR gene was present in 40 patients $(55.6 \%)$ among which there were $11 \mathrm{~T} / \mathrm{T}$ homozygous patients $(15.3 \%$ of the whole studied group).

$66.7 \%$ of the studied group showed disease control during pemetrexed monotherapy, of whom only 7 reached partial response (PR) (9.7\%). In 24 patients (33.3\%) disease progression was observed during first two months after pemetrexed monotherapy. Median progression-free survival reached 4.5 months, while median overall survival reached 9 months.

Surprisingly, among all the analyzed clinical factors, none has significantly influenced the risk of early disease progression or progression-free survival. Slightly prolonged overall survival ( $\mathrm{HR}=0.58, p=0.076$ ) was observed among patients with PFS longer than 6 months after first-line non-pemetrexed chemotherapy (Table 1).

It was observed that among carriers of triple $28 \mathrm{bp}$ tandem repeats with $\mathrm{G}$ allele in the second tandem repeat, disease progression was significantly more frequent $(p=0.0287$ ) than among carriers of other genotype combinations. PFS and OS were shorter in carriers of such genotype, however these were not statistically significant differences (Table 2). Only among carriers of both triple tandem repeats in $T S$ gene and $\mathrm{C} / \mathrm{C}$ genotype in MTHFR $677 \mathrm{C}>\mathrm{T}$ polymorphism, a double shortening of PFS may be observed in comparison to carriers of other genotype combinations. However, this difference was not statistically significant either (Fig. 1, Table 2).

Carriers of both triple $28 \mathrm{bp}$ tandem repeats in both alleles of $T S$ gene and C/C genotype in $677 \mathrm{C}>\mathrm{T}$ SNP in MTHFR gene had significantly shorter overall survival in comparison to carriers of other genotype combinations (6.75 vs. 12 months, $p=0.0031$ ) (Fig. 2, Table 2).

Using Cox logistic regression we were unable to create a model evaluating the power of the impact of the studied clinical and molecular factors on the risk of early progression due to the lack of significance of each determinant. The model created for the risk of early death has reached the significance threshold, however the only variable, which significantly influenced this risk, was the presence of both triple $28 \mathrm{bp}$ tandem repeats in $T S$ gene and $\mathrm{C} / \mathrm{C}$ genotype in $677 \mathrm{C}>\mathrm{T}$ SNP in MTHFR gene as well as short duration of PFS after first-line non-pemetrexed chemotherapy (Table 3).

\section{Discussion}

In second-line therapy, pemetrexed is administered as monotherapy, and this form was registered based on a large-scale, multicenter, randomized, phase III clinical trial performed on 571 NSCLC patients. The study compared the effectiveness of second-line pemetrexed or docetaxel monotherapy and it did not show differences in efficacy of the drugs, however significantly less toxicity was observed after pemetrexed treatment. In pemetrexed arm objective response and stable disease was observed in $71.2 \%$ of the studied group, PFS reached 2.9 months and OS -8.3 months. The study also revealed that the basic clinical factors such as good performance status $(\mathrm{PS}=0)$, locally advanced disease and time since last 


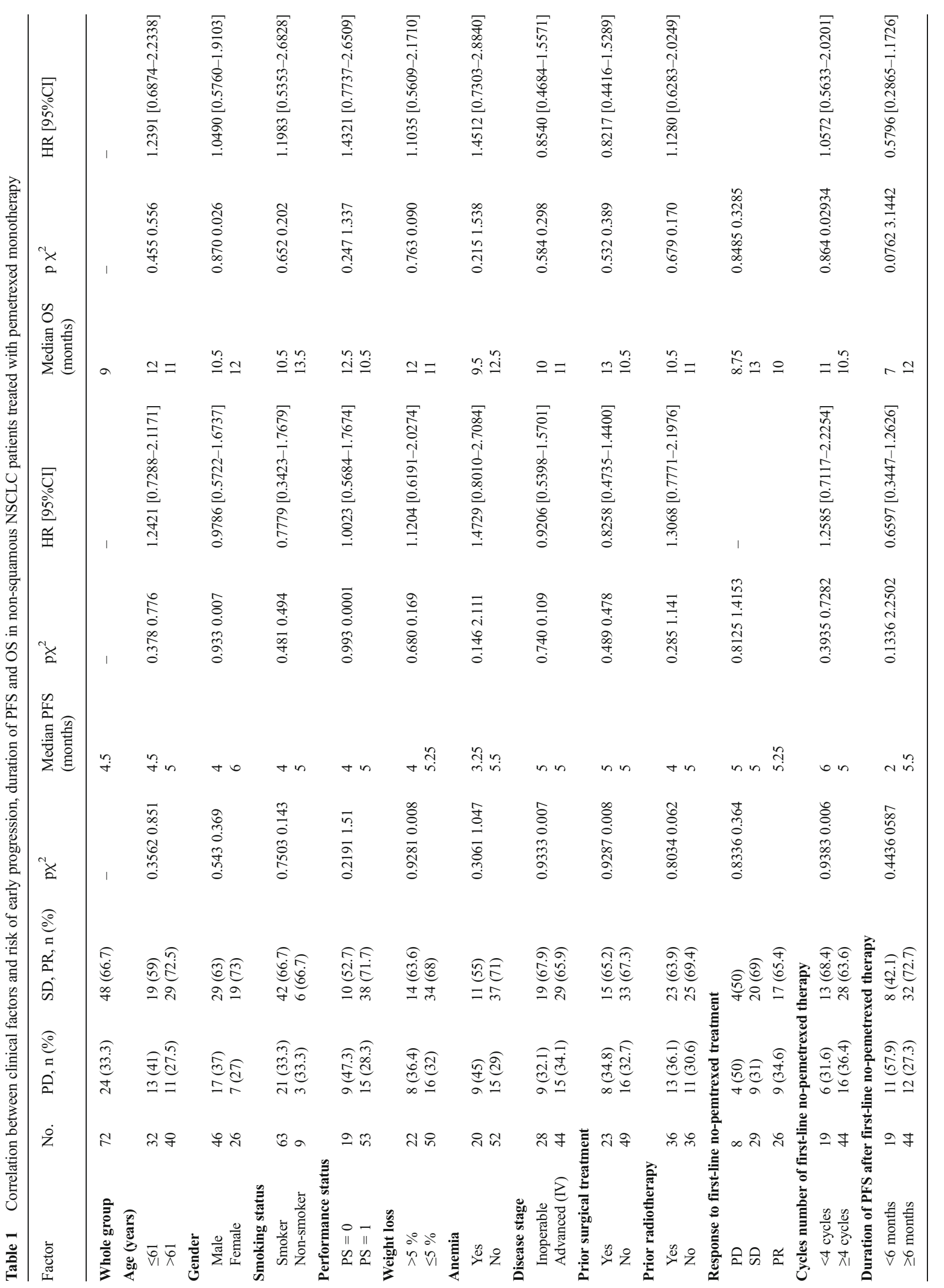




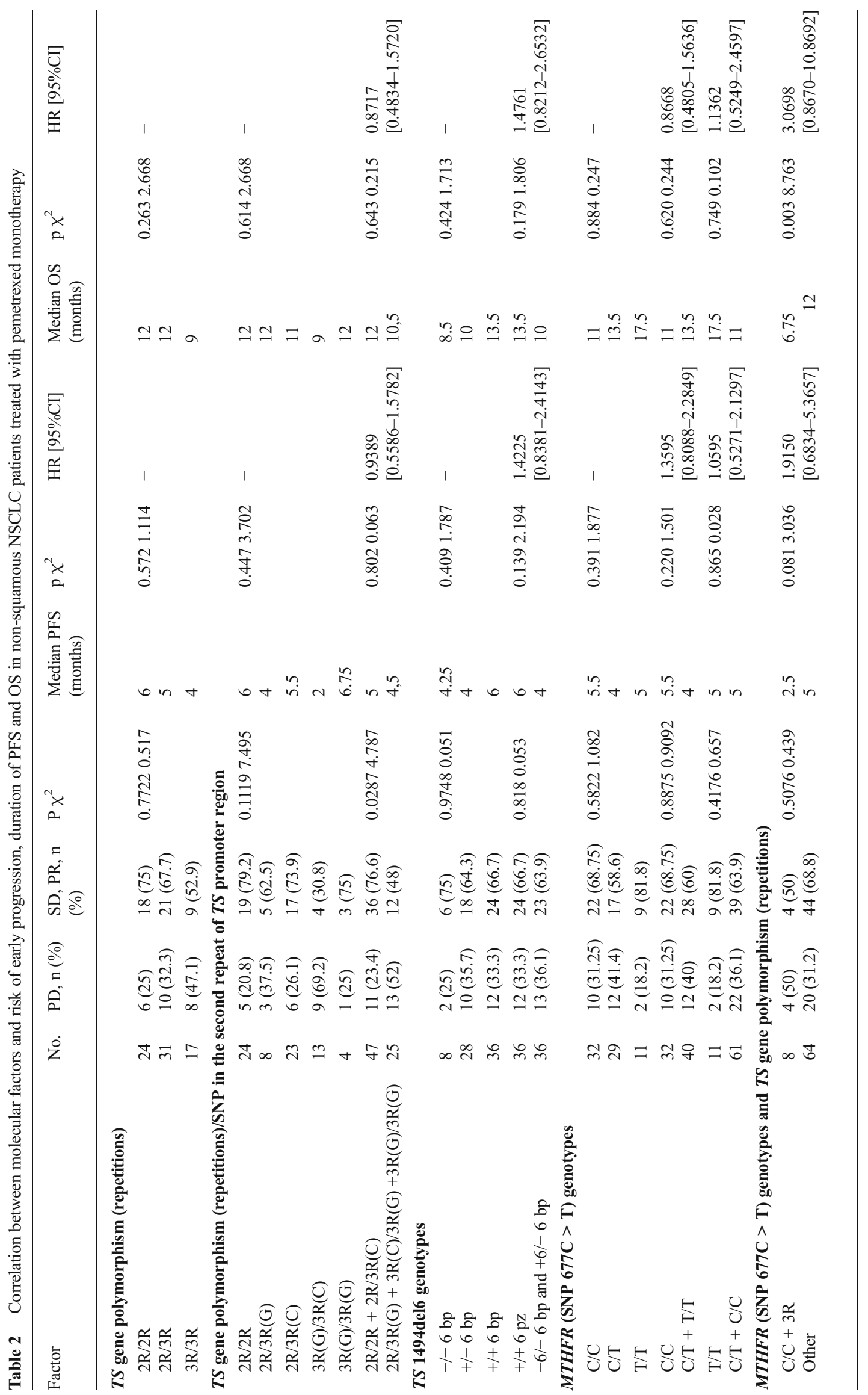


Fig. 1 Impact of TS VNTR polymorphism and a $677 \mathrm{C}>\mathrm{T}$ SNP in MTHFR gene on progression free survival in nonsquamous NSCLC patients treated with pemetrexed monotherapy

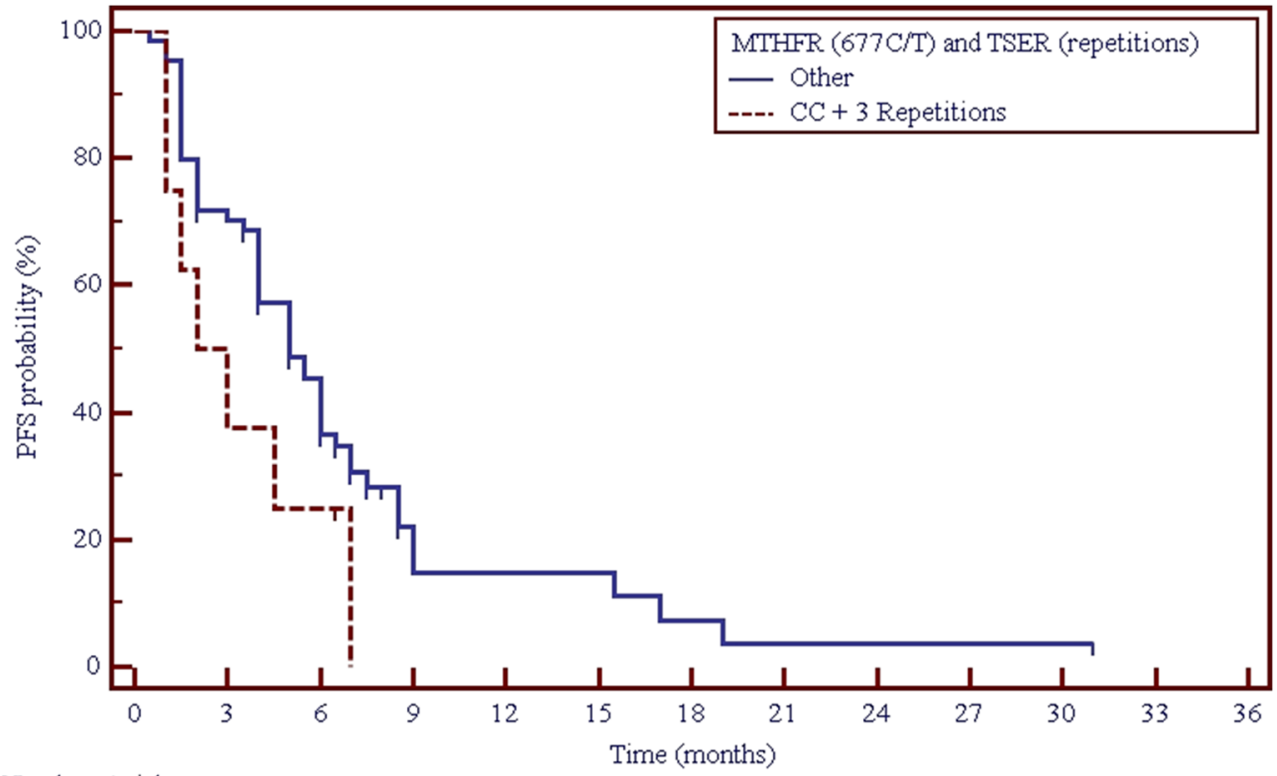

Number at risk

Group: Other

$\begin{array}{llllllllllll}64 & 44 & 20 & 4 & 4 & 4 & 2 & 1 & 1 & 1 & 1 & 0\end{array}$

Group: $\mathrm{CC}+3$ Repetitions chemotherapy over 3 months are positively influencing overall survival in NSCLC patients treated with pemetrexed monotherapy [3].

Pemetrexed monotherapy was compared with a pemetrexed/carboplatin regime, in second-line of NSCLC treatment, in GOIRC 02-2006 clinical trial. PFS in the pemetrexed arm reached 3.6 months and OS -8.8 months, which were similar to the result of the Hanna et al. trial. Twodrug regime showed slightly higher effectiveness but for a price of higher toxicity [19].

Maintenance therapy using pemetrexed is possible in selected non-squamous NSCLC patients. The PARAMOUNT
Fig. 2 Impact of TS VNTR polymorphism and a $677 \mathrm{C}>\mathrm{T}$ SNP in MTHFR gene on overall survival in non-squamous NSCLC patients treated with pemetrexed monotherapy

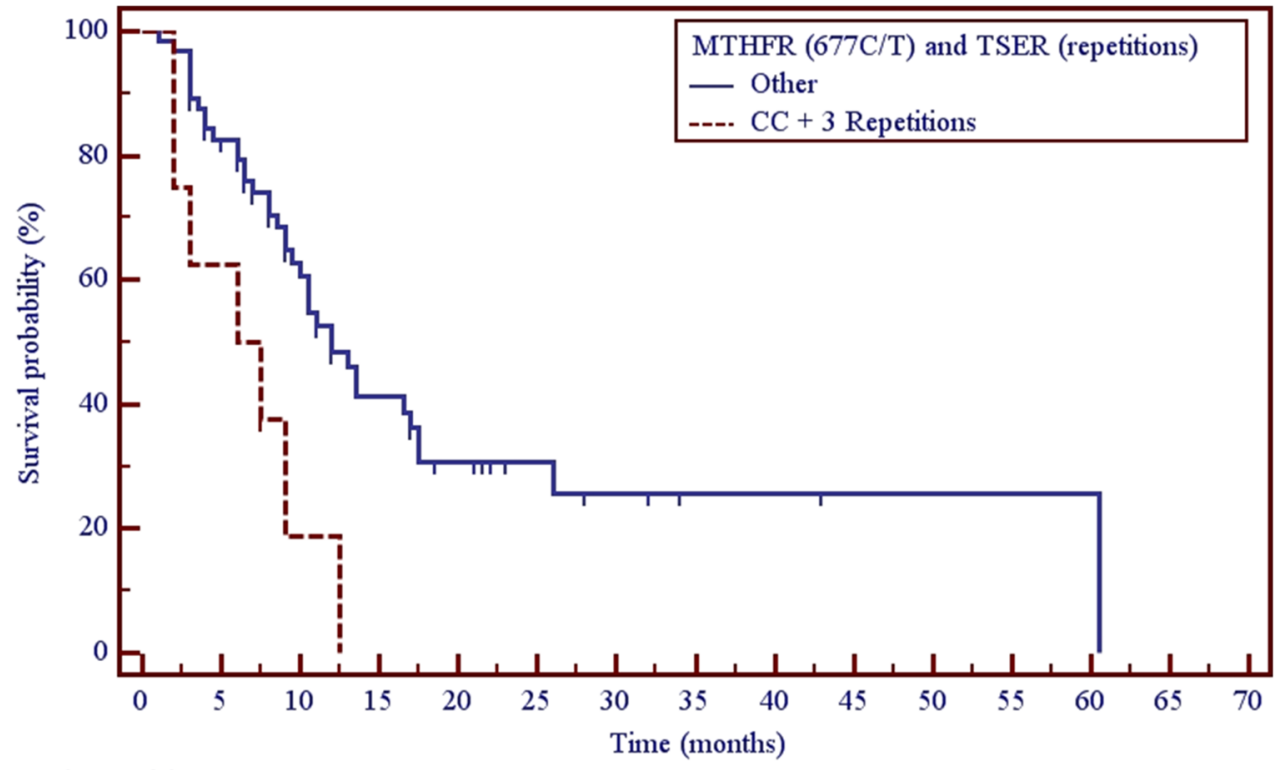

Number at risk

Group: Other

$\begin{array}{cccccccccccccr}64 & 49 & 30 & 17 & 10 & 6 & 4 & 2 & 2 & 1 & 1 & 1 & 1 & 0 \\ \text { Group: } \mathrm{CC}+3 & \text { Repetitions } & & & & & & & & & & & \\ 8 & 5 & 1 & 0 & 0 & 0 & 0 & 0 & 0 & 0 & 0 & 0 & 0 & 0\end{array}$


Table 3 Multivariate Cox logistic regression model of factors affecting the overall survival of non-squamous NSCLC patients treated with pemetrexed monotherapy

\begin{tabular}{llcc}
\hline & $\beta$ & $p$ & $\mathrm{HR}[95 \% \mathrm{CI}]$ \\
\hline C/C genotype of MTHFR SNP 677C $>$ T + 3R/3R genotype of TS gene & 1.3469 & 0.0042 & $3.8456[1.5364-9.6256]$ \\
Short duration of PFS after first-line no-pemetrexed chemotherapy $(<\mathbf{6}$ months) & 0.7385 & 0.00299 & $2.0928[1.0782-4.0622]$ \\
\hline
\end{tabular}

Overall fit model: $p=0.00082, \chi^{2}=9.606$

clinical trial showed that pemetrexed monotherapy after successful first-line pemetrexed/platinum therapy, extends progression-free survival to 4.1 months and overall survival to 13.9 months [20].

In our study, we reached results similar to the ones obtained by Hanna et al. Median PFS in patients treated with pemetrexed monotherapy was 4.5 months and OS - 9 months. Such results were reached even though only $18 \%$ of our studied group received further treatment lines. Only slight but not significant differences in OS are visible between patients with different PS (12.5 vs. 10.5 months for PS $=0$ and $\mathrm{PS}=1$, respectively), in patients with or without anemia (9.5 vs. 12.5 months) and in patients with long and short response to first-line non-pemetrexed chemotherapy (12 vs. 7 months).

Clinical factors are important in proper qualification to chemotherapy but it is not always possible to choose the best option based only on them, thus molecular factors are being studied more and more intensively in order to find relevant ones, also in pemetrexed treatment.

The most often studied molecular factor is the expression of thymidylate synthase. A study on 110 NSCLC patients treated with pemetrexed in third- or fourth-line did not show any significant influence of TS expression on PFS or OS. However it is worth mentioning that the researchers had only limited access to cancer tissue (only 13 out of 55 samples were eligible for immunohistochemical analysis) [21].

Analysis of pemetrexed target genes (TS, DHFR, GARFT and MTHFR) was carried out on 90 adenocarcinoma Asian patients treated with pemetrexed monotherapy in third- or further lines. No relationship was found between the polymorphisms and response rates, but it is interesting that carriers of "high expression" genotypes in TS gene $(2 \mathrm{R} / 3 \mathrm{R}(\mathrm{G}), 3 \mathrm{R}(\mathrm{C}) /$ $3 \mathrm{R}(\mathrm{G}), 3 \mathrm{R}(\mathrm{G}) / 3 \mathrm{R}(\mathrm{G}))$ reached longer PFS (5.2 vs. 3.7 months) and $\mathrm{OS}$ (31.8 vs. 18.5 months) than carriers of "low expression" genotypes $(2 \mathrm{R} / 2 \mathrm{R}, 2 \mathrm{R} / 3 \mathrm{R}(\mathrm{C}), 3 \mathrm{R}(\mathrm{C}) / 3 \mathrm{R}(\mathrm{C}))$, which is in contradiction to the results from the studies of the same polymorphism but in first-line pemetrexed treatment [22].

Smit et al. carried out a comparison of pemetrexed monotherapy with pemetrexed/carboplatin regime in second-line treatment of NSCLC patients. As a part of the study a pharmacogenetic analysis was carried out. There was no connection between "low/high expression" genotypes of TS gene and treatment results, but a significant correlation was observed in carriers of T/T genotype of MTHFR $677 \mathrm{C}>\mathrm{T}$ polymorphism, where PFS was longer for such patients when compared to carriers of other genotypes (7.9 vs. 2.9 months; $p=0.003$ ) [23].

The analysis of two clinical trials: GOIRC 02-2006 (19) and NVALT-7 (23), for polymorphisms connected with pemetrexed effectiveness, showed that T/T genotype of MTHFR $677 \mathrm{C}>\mathrm{T}$ polymorphism is correlated with longer PFS (5.4 vs. 3.4 months; $p=0.012)$ and OS (16.4 vs. 8.5 months; $p=0.026)$ when compared to other genotypes $(\mathrm{C} / \mathrm{C}$ and $\mathrm{C} / \mathrm{T})$ [24].

In our study carriers of "high expression" genotypes in TS gene showed significantly more frequent early progression than carriers of "low expression" genotypes. Small (but not statistically significant) differences in OS were observed among carriers of different genotypes of MTHFR gene $677 \mathrm{C}>\mathrm{T}$ polymorphism (17.5 vs. 11 months for carriers of $\mathrm{T} / \mathrm{T}$ and $\mathrm{C} / \mathrm{C}+\mathrm{C} / \mathrm{T}$, respectively). Among carriers of $3 \mathrm{R} / 3 \mathrm{R}$ genotype in TS gene and C/C genotype of MTHFR $677 \mathrm{C}>\mathrm{T}$ polymorphism a significantly shorter OS was observed $(6.75$ vs. 12 months for carriers of $3 \mathrm{R} / 3 \mathrm{R}+\mathrm{C} / \mathrm{C}$ and carriers of other genotype combinations respectively; $p=0.0031$ ). Moreover, in multivariate analysis, significantly higher risk of death was observed in carriers of both 3R/3R genotype in TS gene and $\mathrm{C} /$ $\mathrm{C}$ genotype in $677 \mathrm{C}>\mathrm{T}$ SNP in MTHFR gene as well as in patients with short PFS after first-line non-pemetrexed chemotherapy. These results are in line with other studies, where the presence of 3R/3R genotype in $T S$ gene and $\mathrm{C} / \mathrm{C}$ genotype in MTHFR gene where considered negative predictive factor in pemetrexed therapy.

Molecular factors become more meaningful when considering a relevant treatment option for cancer patients. Studies carried out on first-line pemetrexed/platinum treated patients seem to confirm usefulness of some factors such as thymidylate synthase expression, but in pemetrexed monotherapy group there is still very little relevant information. It is also worth mentioning that patients who receive second or further lines of chemotherapy usually have worse prognosis than those who are treated with first-line. Therefore, based on our study, it appears that the studied genetic factors may have high predictive and prognostic value (as high as clinical factors). Our results show that analysis of VNTR polymorphism in $T S$ gene, an inside $\mathrm{G}>\mathrm{C}$ SNP in VNTRs and MTHFR gene $677 \mathrm{C}>\mathrm{T}$ SNP might help in selecting a group of non-squamous NSCLC patients who will benefit significantly from pemetrexed monotherapy, but in order 
to evaluate properly the usefulness of the chosen molecular factors more large-scale and prospective studies on more representative groups are needed.

\section{Conflict of Interest Authors declare no conflict of interest.}

Open Access This article is distributed under the terms of the Creative Commons Attribution 4.0 International License (http:// creativecommons.org/licenses/by/4.0/), which permits unrestricted use, distribution, and reproduction in any medium, provided you give appropriate credit to the original author(s) and the source, provide a link to the Creative Commons license, and indicate if changes were made.

\section{References}

1. Dela Cruz CS, Tanoue LT, Matthay RA (2011) Lung cancer: epidemiology, etiology, and prevention. Clin Chest Med 32:605-644

2. Scagliotti GV, Parikh P, von Pawel J, Biesma B, Vansteenkiste J, Manegold C, et al. (2008) Phase III study comparing cisplatin plus gemcitabine with cisplatin plus pemetrexed in chemotherapy-naive patients with advanced-stage non-small-cell lung cancer. J Clin Oncol 26:3543-3551

3. Hanna N, Shepherd FA, Fossella FV, Pereira JR, De Marinis F, von Pawel J, et al. (2004) Randomized phase III trial of pemetrexed versus docetaxel in patients with non-small-cell lung cancer previously treated with chemotherapy. J Clin Oncol. 22:1589-1597

4. Paz-Ares LG, de Marinis F, Dediu M, Thomas M, Pujol JL, Bidoli P, et al. (2013) PARAMOUNT: final overall survival results of the phase III study of maintenance pemetrexed versus placebo immediately after induction treatment with pemetrexed plus cisplatin for advanced nonsquamous non-small-cell lung cancer. J Clin Oncol. 31:2895-2902

5. Chen CY, Chang YL, Shih JY, Lin JW, Chen KY, Yang CH, et al. (2011) Thymidylate synthase and dihydrofolate reductase expression in non-small cell lung carcinoma: the association with treatment efficacy of pemetrexed. Lung Cancer 74:132-138

6. Buque A, Aresti U, Calvo B, Sh Muhialdin J, Munoz A, Carerra S, et al. (2013) Thymidylate synthase expression determines pemetrexed targets and resistance development in tumour cells. PLoS One 8:e63338

7. Wang T, Chuan Pan C, Rui Yu J, Long Y, Hong Cai X, De Yin X, et al. (2013) Association between TYMS expression and efficacy of pemetrexed-based chemotherapy in advanced non-small cell lung cancer: ameta-analysis. PLoS One 10(8):e74284

8. Ceppi P, Volante M, Saviozzi S, Rapa I, Nevello S, Cambieri A, et al. (2006) Squamous cell carcinoma of the lung compared with other histotypes shows higher messenger RNA and protein levels for thymidylate synthase. Cancer 107:1589-1596

9. Tanaka F, Wada H, Fukui Y, Fukushima M (2011) Thymidylate synthase (TS) gene expression in primary lung cancer patients: a large-scale study in Japanese population. Ann Oncol 22:1791-1797

10. Tiseo M, Giovannetti E, Tibaldi C, Camerini A, Di Costanzo F, Barbieri F, et al. (2012) Pharmacogenetic study of patients with advanced non-small cell lung cancer (NSCLC) treated with second-line pemetrexed or pemetrexed-carboplatin. Lung Cancer 78:92-99

11. Horie N, Aiba H, Oguro K, Hojo H, Takeishi K (1995) Functional analysis and DNA polymorphism of the tandemly repeated sequences in the 5 '-terminal regulatory region for the human gene thymidylate synthase. Cell Struct Funct 20:191-197

12. Mandola MV, Stoehlmacher J, Muller-Weeks S, Cesarone G, Yu MC, Lenz HJ, et al. (2003) A novel single nucleotide polymorphism within the 5 ' tandem repeat polymorphism of the thymidylate synthase gene abolishes USF-1 binding and alters transcriptional activity. Cancer Res 63:2898-2904

13. Mandola MV, Stoehlmacher J, Zhang W, Groshen S, Yu MC, Iqbal S, et al. (2004) A 6 bp polymorphism in the thymidylate synthase gene causes message instability and is associated with decreased intratumoral TS mRNA levels. Pharmacogenetics 14:319-327

14. Sulzyc-Bielicka V, Bielicki D, Binczak-Kuleta A, Kaczmarczyk M, Pioch W, Machoy-Mokrzynska M, et al. (2013) Thymidylate synthase gene polymorphism and survival of colorectal cancer patients receiving adjuvant 5-fluorouracil. Gent Test Mol Biomarkers 17: 799-780

15. Sohn KJ, Croxford R, Yates Z, Lucock M, Kim YI (2004) Effect of the methylenetetrahydrofolate reductase $\mathrm{C} 677 \mathrm{~T}$ polymorphism on chemosensitivity of colon and breast cancer cells to 5-fluorouracil and methotrexate. J Natl Cancer Inst 96:134-144

16. Iacopetta B, Grieu F, Joseph D, Elsaleh H (2001) A polymorphism in the enhancer region of the thymidylate synthase promoter influences the survival of colorectal cancer patients treated with 5-fluorouracil. Br J Cancer 85:827-830

17. Dotor E, Cuatrecases M, Martínez-Iniesta M, Navarro M, Vilardell F, Guinó E, et al. (2006) Tumor thymidylate synthase 1494del6 genotype as a prognostic factor in colorectal cancer patients receiving fluorouracil-based adjuvant treatment. J Clin Oncol 24:16031611

18. Frosst P, Blom HJ, Milos R, Goyette P, Sheppard CA, Matthews $\mathrm{RG}$, et al. (1995) A candidate genetic risk factor for vascular disease: a common mutation in methylenetetrahydrofolate reductase. Nat Genet 10:111-113

19. Ardizzoni A, Tiseo M, Boni L, Vincent AD, Passalacqua R, Buti S, et al. (2012) Pemetrexed versus pemetrexed and carboplatin as second-line chemotherapy in advanced non-small-cell lung cancer: results of the GOIRC 02-2006 randomized phase II study and pooled analysis with the NVALT7 trial. J Clin Oncol 30:4501-4507

20. Paz-Ares L, de Marinis F, Dediu M, Thomas M, Pujol JL, Bidoli P, et al. (2012) Maintenance therapy with pemetrexed plus best supportive care versus placebo plus best supportive care after induction therapy with pemetrexed plus cisplatin for advanced non-squamous non-small-cell lung cancer (PARAMOUNT): a double-blind, phase 3, randomized controlled trial. Lancet Oncol 13:247-255

21. Chang MH, Ahn JS, Lee J, Kim KH, Park YH, Han J, et al. (2010) The efficacy of pemetrexed as a third- or fourth-line therapy and the significance of thymidylate synthase expression in patients with advanced non-small cell lung cancer. Lung Cancer 69:323-329

22. Jung M, Lee CH, Park HS, Lee JH, Kang YA, Kim SK, et al. (2013) Pharmacogenomic assessment of outcomes of pemetrexed-treated patients with adenocarcinoma of the lung. Yonsei Med J 54:854 864

23. Smit EF, Burgers SA, Biesma B, Smit HJ, Eppinga P, Dingemans AM, et al. (2009) Randomized phase II and pharmacogenetic study of pemetrexed compared with pemetrexed plus carboplatin in pretreated patients with advanced non-small-cell lung cancer. J Clin Oncol 27:2038-2045

24. Tiseo M, Giovannetti E, Tibaldi C, Camerini A, Di Costanzo F, Barbieri F, et al. (2012) Pharmacogenetic study of patients with advanced non-small cell lung cancer (NSCLC) treated with second-line pemetrexed or pemetrexed-carboplatin. Lung Cancer 78:92-99 